\title{
Los gremios medievales de los agricultores y sus estatutos en las tierras pontificias (siglos XIV y XV)
}

\author{
The medieval guilds of farmers and their statutes in the pontifical \\ lands (14th-15th centuries)
}

Simone ROSATI

\begin{abstract}
Author:
Simone Rosati

Profesor de Derecho romano y de Historia del Derecho. Facultad de Ciencias Humanas, Canónicas y Religiosas. Universidad Católica de Murcia (Murcia, Spain)

srosati@ucam.edu

https://orcid.org/0000-0002-2522-4399

Date of reception: 31/03/21

Date of acceptance: 07/07/21

\section{Citation:}

Rosati, S. (2021). Los gremios medievales de los agricultores y sus estatutos en las tierras pontificias (siglos XIV y XV). Anales de la Universidad de Alicante. Historia Medieval, (22), 341-361.

https://doi.org/10.14198/medieval.19538

(C) 2021 Simone Rosati

Licence: This work is licensed under a Creative Commons Attribution 4.0 International License (CC BY 4.0).
\end{abstract}

\section{RESUMEN}

El presente estudio pretende reconstruir la explotación y gestión de los recursos naturales en los territorios pontificios en los siglos XIV y XV. La investigación se llevará a cabo a través del estudio histórico-jurídico de los testimonios dejados por las corporaciones entre los agricultores en los dominios temporales de la Iglesia. Tras describir las peculiaridades gremiales agrícolas medievales y sus estatutos, se examinarán con detalle los cuatro gremios de agricultores que, en el estado actual de la investigación, están presentes en la zona geográfica considerada: Tarquinia, Viterbo, Tuscania y Roma. Las tres primeras corporaciones serán objeto de un estudio común ya que presentan considerables similitudes tanto en contenido como estructura, que permiten suponer una influencia mutua en la redacción de los iura propria. El gremio de Roma, que constituye un unicum en el panorama italiano, será objeto de un estudio específico con el fin de representar sus elementos originales.

El examen de las fuentes citadas permitirá comprender no sólo la organización interna de los gremios medievales de los agricultores y el tipo de actividad desarrollada en relación con la gestión de los recursos naturales, sino que también ofrecerá una visión más amplia del sistema de propiedad 
medieval, de la administración de justicia en las tierras pontificias y de la relación entre ius commune e iura propria.

PALABRAS CLAVE: gremios medievales; agricultores; estatutos medievales; Estado Pontificio.

\section{ABSTRACT}

The present study intends to reconstruct the exploitation and management of natural resources in the papal territories in the 14th and 15th centuries. The research will be carried out through the historical-legal study of the precious testimonies left by the corporations among farmers in the temporal dominions of the Church. After describing the peculiarities of medieval agricultural associations and their statutes, an examination of the four guilds among farmers that, at the current state of research, are present in the geographical area under consideration will be proposed: Tarquinia, Viterbo, Tuscania, and Rome. The first three corporations will be treated together since they present considerable similarities in content and structure, which allows us to hypothesize a mutual influence in the drafting of the iura propria. The corporation of Rome, which is unique in Italy, will be the object of a specific study. The study of these sources will not only provide an insight into the internal organisation of the medieval farmers' guilds and the type of activity carried out in relation to the management of natural resources, but will also give a broader view of the medieval property system, the administration of justice in the Papal lands and the relationship between ius commune and iura propria.

Keywords: medieval corporations; farmers; medieval statutes; Papal State.

\section{LOS GREMIOS DE AGRICULTORES EN LA EXPERIENCIA JURÍDICA MEDIEVAL}

Ya en la época romana se ha constatado la existencia de los primeros gremios que agrupaban personas que ejercían un mismo oficio bajo el nomen de collegia opificium; estos surgieron primero por libre iniciativa de los trabajadores que se agrupaban en un mismo sector económico, aunque posteriormente, en la época imperial y bizantina, se convirtieron directamente en instituciones controladas por el Estado (Caroselli, 1957, p. 864; Arangio Ruiz, 2006, p. 71; Diosono, 2007; De Robertis, 1971, 1981; Kloppenbog-Wilson, 2002; Flambard, 1981; Mennella-Apicella, 2000; Patterson, 1993, 1994).

En los antiguos pueblos germánicos también existían los denominados gilde, regidos por una asamblea en la que se discutían los asuntos generales del mismo (Calasso, 1949, 1954; Leicht, 1937). Este fenómeno asociativo gremial, de tan vasto alcance, ha sido objeto de numerosos estudios de los que, sobre todo en el pasado, una parte conspicua se ha dirigido a buscar su origen en el mundo germánico o en 
el romano. En realidad, si estas organizaciones de profesiones anteriores tuvieron cierta influencia en los gremios medievales, hay que considerar primero que cada periodo histórico responde a esquemas, normas y necesidades diferentes (Bezzina, 2013).

Así, los collegia romanos, como instituciones estatales rígidamente insertadas en la estructura administrativa imperial, no son en absoluto comparables a las corporaciones gremiales medievales, ni los gremios del mundo germánico tuvieron un peso decisivo en el nacimiento del asociacionismo en la Edad Media. Se trata, por tanto, de un fenómeno original que se inserta en un contexto social y económico completamente nuevo que, a partir del siglo XII, involucra a toda Europa (Calasso, 1949, pp. 132-139).

En Europa, y concretamente en Italia, los gremios medievales fueron el ejemplo más representativo de un momento histórico de renacimiento cultural, económico y social que se originó en la ciudad con todas sus vivas articulaciones sociales. Cualquier investigación que busque el significado auténtico de los gremios medievales no podrá prescindir nunca del análisis del contexto de la ciudad, pues la historia de los municipios es en esencia la historia de las asociaciones gremiales que surgieron en ellos. Uno de los rasgos más peculiares de los gremios medievales, es que, al ser una parte esencial de los asuntos de la ciudad, participan activamente en la vida política, religiosa y económica del municipio, por lo que son en realidad entidades de carácter político (Black, 1984, 1990; Zanoboni, 2009; Braunstein, 2003, 2007; Coornaert, 1947, pp. 208-243; Thrupp, 1965, pp. 230-280; Sprandel, 1991, pp. 353-366).

En lo que respecta a los gremios agrícolas de la Edad Media, se pueden distinguir dos tipos de asociaciones (Rasi, 1940, pp. 7-9):

- Los gremios nacidos para la explotación de la propiedad indivisa.

- Los gremios de propietarios o trabajadores de la tierra.

En el primer caso, prevalece el vínculo real, dado por la ingente propiedad indivisa de los señores locales o de los eclesiásticos que dejaban cuotas de tierra para su disfrute, o en otros casos por la propiedad pública del municipio entregada a los ciudadanos para su uso (como es el caso del ager publicus romano), o incluso por la propiedad, en régimen de propiedad colectiva, de un determinado grupo nobiliario o comunidad de habitantes.

Así que las asociaciones se formaron para el disfrute de la propiedad indivisa con el fin de garantizar una distribución equitativa de las tierras comunes en las que se practicaba principalmente el pastoreo. Numerosas disposiciones, tanto consuetudinarias como estatutarias, regulaban las relaciones entre los vicini, que debían respetar y ayudar a los demás miembros; otras normas detalladas estaban previstas para las reuniones periódicas, la resolución de los conflictos, la fiesta en honor del santo patrón, el uso de las tierras comunales y las funciones de los oficiales (porcherii, casarii, caprarii) que tenían la tarea de dirigir los rebaños de los vecinos. 
En el segundo caso, el de las asociaciones entre propietarios o trabajadores de la tierra, predomina la relación personal entre los miembros de una categoría específica de pequeños propietarios o arrendatarios de parcelas agrícolas que buscan defenderse de las demás asociaciones rurales y artesanales del municipio; esta forma de asociación, a diferencia de la primera, asume en muchos casos el carácter de persona jurídica, teniendo los requisitos constitutivos como la voluntas societatis, un patrimonio, una finalidad, un vínculo social y una jurisdicción propia.

Los dos tipos de asociaciones entre agricultores descritos anteriormente tienen la estructura organizativa típica de los gremios medievales, aunque con variaciones en la terminología o en la administración, debido principalmente a las costumbres locales.

Las asociaciones, denominadas scholae, fraglie, paratici, arti e mestieri, universitates o fraternites, estaban gobernadas por uno o varios jefes (rectores, cónsules, capitanes, podestà), que ejercían funciones políticas, ejecutivas y judiciales; todos los miembros solían reunirse en asambleas generales, durante las cuales se tomaban las decisiones más importantes y se elegían los cargos, mientras que un número limitado de jurados constituía el consejo del gremio, que se encargaba, entre otras funciones, de proteger los intereses de la sociedad, aprobar los estatutos e interpretar las normas; por último, el aparato administrativo del gremio incluía a los distintos oficiales como los tesoreros, los notarios y las figuras específicas de cada sector económico; común a las corporaciones de oficios medievales era también el sentimiento religioso y el espíritu mutualista entre los miembros (Lattes, 1884; Artifoni, 1988, pp. 9-40; Occhipinti, 1990, pp. 101-174; Canning, 1980, pp. 9-32; Degrassi, 1996, 2000, pp. 17-35, 2001, pp. 147-173, 2010, pp. 273-287; Greci, 1995, pp. 61-106; Pini, 1976, 1986; Tamassia, 1898, pp. 112-141).

\section{LOS ESTATUTOS DE LOS GREMIOS DE AGRICULTORES EN EL SISTEMA DEL DERECHO COMÚN}

La expresión derecho común representa las dos grandes almas del derecho bajo medieval: el jus commune y el jus proprium. El primero (jus commune) identifica no sólo el derecho imperial justinianeo que el maestro Irnerio recompuso e hizo utilizable, sino también el Derecho canónico, realizando así el mecanismo del utrumque jus en el que "uno y otro derecho" están íntimamente coordinados (Calasso, 1954, pp. 526-528; Bellomo, 2016; Wolf, 1993-1994, pp. 35-41; Montanos Ferrín, 2007, pp. 469-479; Minnucci, 2017, pp. 77-106).

En la mentalidad medieval la lex mundana era expresión de la unidad política constituida por el emperador, la lex ecclesiastica, símbolo de la unidad espiritual, emanaba del Pontífice, pero, al mismo tiempo, ambas jurisdicciones tenían como único destinatario a la persona, tanto creyente como ciudadana (Calasso, 1954, p. 407). 
El segundo (jus proprium) incluye el ius de los ordenamientos particulares tanto de derecho civil como de derecho canónico, como la costumbre y los estatutos de los municipios o de las corporaciones. A pesar de la gran fuerza práctica y política del jus proprium, sólo en el siglo XIV los juristas medievales, tenazmente apegados a la universalidad e inmovilidad del derecho imperial, se rindieron ante la realidad siempre cambiante de la vida y reconocieron su dignidad jurídica (Campitelli, 2000, pp. 42-44).

A este respecto, Bártolo de Sassoferrato proporcionó una clara explicación de los límites del ius statuendi de las corporaciones de oficios: “...collegia licita et approbata in his in quibus habent iurisdictionem et quo ad ea quae ad ipsos collegiatos pertinent, possunt facere statuta..." (Bartoli, ad D. 1,1 de iustitia et iure, 1. 9 omnes populi, n. 6).

Otra cuestión tratada por los maestros del Derecho común fue la relación entre el ius proprium de los gremios y el de los municipios, que, como es sabido, no siempre fue pacífica. Según Alberico De Rosate, era deseable que hubiera concordancia entre ambas fuentes, pero al mismo tiempo no descartaba la posibilidad de que los estatutos corporativos, aunque estuvieran en contraste con los municipales, mantuvieran su eficacia frente a los miembros: “...Collegia licita possunt facere statuta...dummodo non controdicant iuri divino vel naturali vel etiam iuri communi...et idem nisi controdicerent statutis civitatis cui subsunt...Credo tamen inter eos quoad eorum praeiudicium tenere..." (Albericus De Rosate, De statutis, I, 6).

Baldo, en cambio, creía que era necesario desaconsejar enérgicamente tales discordancias entre categorías de estatutos porque "diversitates sunt odiosae et plenae tristissimi eventus..." (Baldi, ad D. 1, 1 de iust. Et iure, 1. 9 omnes populi n. 32).Una última diatriba sobre los estatutos corporativos se refiere a su confirmación por parte del municipio, el maestro Baldo afirmó que "...ista statuta sunt confirmata a iure communi, et ideo non est nocesse quod confirmentur per legem municipalem..." (Baldi, ad C. 4, 18 de const. pec. 1. 2 recepticia n. 9). Bartolomeo de Saliceto sostenía una opinión diferente, la confirmación de la civitas era un elemento deseable y útil, ya que permitía extender el contenido preceptivo del estatuto a sujetos y personas generalmente exorbitantes de los límites de esa fuente (Schioppa, 1974, pp. 200-201).

En cuanto al contenido de los estatutos corporativos, éstos recogen el ordenamiento de una determinada corporación de oficios en el que confluyen las costumbres locales, fuente de conexión y el préstamo entre el ius proprium civile y el ius proprium canónico, los juramentos de los miembros o de sus representantes y las deliberaciones del órgano que ostenta el poder legislativo; un proceso similar de positivización se encuentra también en los estatutos municipales.

Todo este material normativo, recogido en la compilación estatutaria a menudo de forma confusa y asistemática, por comodidad expositiva, se remonta a dos grandes temas: uno de carácter interno y otro de carácter externo al gremio.

El primero, de carácter interno, se refiere a las relaciones entre los miembros del gremio y está destinado a establecer normas de conducta no sólo puramente 
jurídico-administrativas, sino también morales y religiosas, como demuestran las disposiciones relativas a las celebraciones de los difuntos, la asistencia a los enfermos, el respeto entre los miembros, las procesiones en honor de los santos. Este es probablemente el ámbito más antiguo de los estatutos gremiales, en el que tienen cabida antiguas costumbres locales cristalizadas a través de comportamientos prolongados en el tiempo, mantenidos por una comunidad con la intención psicológica de darse una norma.

La segunda, de carácter externo, se basa en la relación entre el gremio y el municipio, una cuestión extremadamente delicada ya que las dos fuentes del ius proprium debían evitar invadir sus respectivas esferas de competencia.

Por este motivo, se estableció la confirmación de los estatutos de las corporaciones de oficios por parte de las autoridades municipales, un episodio que confirma la voluntad de evitar que la persecución de los intereses particulares de la corporación causase perjuicios al municipio (Besta, 1925, p. 686). Además, los estatutos, si procedían de municipios de los Estados Pontificios, como en el caso que se analiza, siempre comenzaban con expresiones de obediencia y lealtad a la Iglesia e incluían la aprobación de los estatutos por parte de la autoridad eclesiástica, con la reserva de que cualquier disposición contraria al Papa sería anulada (Dani, 2012, pp. 4-5).

En general, éste era el contenido de los estatutos corporativos, que eran elaborados por comisiones de técnicos, los statutarii en muchos casos carentes de la habilidad de los juristas, pero sensibles a las necesidades prácticas de los gremios. Dado que estas obras normativas se dirigían al pueblo y debían adaptarse a la vida cotidiana, la sublime lengua latina, incomprensible para el hombre común, dio paso a la lengua vernácula.

En la Baja Edad Media, hubo muchas civitates en las que los agricultores se organizaron según estatutos que no se dirigían indistintamente a todos los agricultores, sino a clases específicas campesinos, como los trabajadores de los castañares, los viticultores, los comerciantes agrícolas o los ganaderos (Cortonesi-Viola, 2006; Rasi, 1940, 100-104).

Un caso peculiar en la historia de los gremios entre agricultores lo representa los Statuta Bobacteriorum Urbis de Roma, dirigidos a todos los propietarios, pecudiarii, jornaleros, comerciantes de productos agrícolas, ganaderos, independientemente de su pertenencia al gremio respectivo (Ricci, 1893, pp. 131-180).

En el siguiente apartado se examinarán los estatutos de las corporaciones entre los agricultores de los Estados Pontificios. En particular, en el apartado 3 se reconstruirá el derecho de las corporaciones agrícolas de tres municipios pertenecientes a la circunscripción territorial del Patrimonio de San Pedro en Tuscia, mientras que en el apartado 4 se examinarán los estatutos de los agricultores de Roma que constituyen un unicum respecto a otras asociaciones entre agricultores. 
Los estatutos examinados muestran no sólo el tipo de actividad agrícola desarrollada en los territorios pontificios, sino también su configuración jurídica basada en una delicada relación de complementariedad entre la pequeña propiedad privada y la propiedad comunitaria (Dondarini, 2006, pp. 115-132; Cristoferi, 2016, pp. 577604).

El punto de equilibrio entre las dos formas de explotación de los recursos naturales venía dado por la complementariedad entre la práctica del cultivo y el pastoreo: mientras que las tierras cultivadas no permitían el pastoreo, que habría destruido sus frutos, los campos no cultivados estaban a libre disposición de los ciudadanos para el ejercicio de los derechos colectivos de pastoreo (ius pascendi) (Dani, 2013; Rosati, 2018).

Así, junto a la propiedad comunitaria generalmente reservada para el pastoreo, existía la propiedad individual destinada a los cultivos de valor y concentrada en el perímetro de las ciudades, para facilitar el transporte de mercancías y, sobre todo, el suministro de agua. Este era el ámbito de actuación de "los estatutos de los ortolani" en los que se establecía una estricta normativa que regulaba únicamente la práctica del cultivo en la pequeña propiedad agrícola.

Además de la relación entre campos cultivados y no cultivados, se daba también el fenómeno típicamente medieval de la fragmentación del dominio en directo y útil. De este modo, era posible que diferentes titulares (soberanos, privados, municipios, señores feudales) tuvieran acceso a la misma parcela en función de las diferentes utilidades que la tierra podía proporcionar. Por ejemplo, en el mismo terreno el derecho de pastoreo (ius pascendi) podía tener un titular diferente al que disfrutaba del derecho de siembra (ius serendi) o de leña (ius lignandi).

Era un sistema especialmente complejo y alejado de la forma actual de entender la actividad productiva sobre la tierra, pero reflejaba muy bien la vida cotidiana de aquellos tiempos (que se conservaron al menos hasta finales del siglo XIX), cuando la población que vivía en un municipio era muy inferior a la que podía trabajar de forma intensiva toda la superficie de la localidad. Es comprensible por qué en aquella época el cultivo se concentraba en las cercanías de la ciudad, mientras que las inmensas extensiones que la rodeaban, a menudo sometidas a condiciones climáticas insalubres, se dejaban en manos de los rebaños.

El vínculo con la ciudad, además, era especialmente evidente ya que estos gremios, a pesar de su vocación agrícola, desarrollaban muchas de sus actividades en la ciudad (como en el caso del mercado de frutas y verduras), solían reunirse en una iglesia y participaban en los principales acontecimientos económicos, políticos y religiosos de la misma. 


\section{LOS GREMIOS AGRÍCOLAS EN EL PATRIMONIO DE SAN PEDRO EN TUSCIA Y SUS ESTATUTOS}

El "Patrimonio de San Pedro en Tuscia" identifica un distrito administrativo establecido por Inocencio III (1198-1216), que incluye las tierras bajo dominio papal situadas en los territorios al norte de Roma. En 1227 Honorio III estableció los límites naturales del Patrimonio de San Pedro, que se extendían "a Radicofano usque Romam", es decir, entre los ríos Tíber, Paglia, Fiora y el mar Tirreno (Paravicini Bagliani, 2010, pp. 668-669; Ferreri, 2018, pp. 1-42).

Los municipios más significativos de esta provincia pontificia eran Orvieto, Orte, Montefiascone, Viterbo, Toscanella (Tuscania), Civita Castellana, Sutri y Nepi, con Corneto (Tarquinia) y Civitavecchia en el lado del mar.

Precisamente en Tarquinia, Viterbo y Tuscania se conservan los estatutos de los gremios de agricultores, conocidos como los "Statuti de li ortolani", que presentan una notable coincidencia estructural y de contenido que sugiere un análisis común (Giontella, 1971-1972; Cuturi, 1883; Rosati, 2013-2014, 2018).

De hecho, en los tres códigos agrarios, las normas sobre el uso del agua para el riego, el mercado de frutas y verduras, las fiestas religiosas, la ayuda mutua entre los miembros y los cargos electivos presentan considerables similitudes. Aunque no es posible establecer cuál de las tres fuentes sirvió de modelo, no cabe duda de que la proximidad geográfica de los tres municipios de la Tuscia papal favorecieron la influencia mutua en la redacción de los textos.

En particular, en los tres estatutos examinados, los legisladores prestan especial atención a dos cuestiones: el riego y la gestión del mercado, se trataba de asuntos que necesitaban una regulación precisa, ya que implica a los agricultores y a sus respectivos intereses económicos.

De hecho, es fácil imaginar las disputas que podían surgir entre los vecinos por la recogida de agua para el riego, bien por los intentos de desviar el curso de las acequias a su favor, o bien por la elección de los puestos en el mercado de la ciudad o la forma de llevar a cabo las negociaciones con los clientes. Por lo tanto, eran necesarias normas basadas en los principios de imparcialidad y objetividad para que nadie sospechara que las normas favorecían a un miembro en detrimento de otro.

Los estatutos agrícolas de Tarquinia, Viterbo y Tuscania fueron redactados respectivamente en 1379, 1481 y 1422, como se deduce del prefacio de los tres códices, las versiones que han llegado hasta nosotros constituyen la reforma de una compilación anterior de la que se ha conservado poca e incompleta información.

\subsection{Estructura organizativa de los gremios de agricultores}

Las asociaciones gremiales de los campesinos de Tarquinia, Viterbo y Tuscania presentan una estructura organizativa casi idéntica, los primeros capítulos de los 
estatutos describen cuales eran las magistraturas corporativas, cómo se elegían, sus atribuciones y deberes (Statuto dell'arte degli ortolani di Tarquinia, 1379, Cap. II, XII, XIII; Statuto dell'arte degli ortolani di Viterbo, 1481, Rubr. V, XI, LVII; Statuta hortulanorum Tuscanae, 1422, Rubr. IV, VII).

Al frente del gremio estaban los rectores asistidos por el camarlengo, los consilgieri, abogados y notarios. Los rectores, en número de 2 o 4, tenían asignadas funciones políticas, ejecutivas, judiciales y asistenciales (Statuto dell'arte degli ortolani di Tarquinia, 1379, Cap. II; Statuto dell'arte degli ortolani di Viterbo, 1481, Rubr. V; Statuta hortulanorum Tuscanae, 1422, Rubr. VI):

- La primera se deriva del hecho de que los dos magistrados eran los representantes del gremio.

- Las segundas atribuciones (función ejecutiva) se referían a la facultad de los rectores de hacer cumplir los estatutos, obligando a los compañeros a prestar juramento sobre las nuevas disposiciones, a pedir al Tesorero los ingresos y gastos de la sociedad, a tomar medidas contra los miembros, a convocar las asambleas de la corporación.

- La tercera categoría de funciones (es decir, las judiciales) se refería a la facultad de los rectores de resolver los conflictos entre los miembros sobre asuntos de competencia de la corporación (riego y mercados de frutas y verduras).

Los rectores también cumplían funciones asistenciales, debiendo socorrer al compañero enfermo que no podía trabajar su huerto o que había sufrido daños en sus tierras, o incluso procurar a costa del gremio un defensor al agricultor que se encontraba injustamente en litigio con el propietario del fundo por motivos relacionados con la renta o cualquier otra razón.

Otro oficial era, como se ha dicho, el camarlengo, que ejercía el mandato de ecónomo y tesorero de la corporación (Statuto dell'arte degli ortolani di Viterbo, 1481, Rubr. XI; Statuta hortulanorum Tuscanae, 1422, Rubr. VII; Statuto dell'arte degli ortolani, 1379, Cap. XII). El camarlengo debía registrar los ingresos y gastos del gremio, custodiar los bienes adscritos a los compañeros, conservar y administrar los bienes de la corporación también mediante un depósito congruente, dar cuenta de su cargo ante todo el colegium.

Otro órgano, esta vez colegiado, era el consejo, compuesto por los jurados cada uno de un distrito diferente del municipio (Statuto dell'arte degli ortolani di Tarquinia, 1379, Cap. XIII; Statuto dell'arte degli ortolani di Viterbo, 1481, Rubr. LVII; Statuta hortulanorum Tuscanae, 1422, Rubr. VI). Los consejeros debían asistir a las reuniones del consejo cuya función era velar por los intereses de la corporación; también debían reformar los estatutos o participar en la redacción de los nuevos junto con los rectores y el Tesorero.

Fundamentales para el ejercicio de las funciones judiciales era la figura del notario, que debía protocolizar las actas, custodiar los archivos de la corporación y 
participar en la reforma de los estatutos y el abogado para representar los intereses del colegium en caso de litigio (Statuto dell'arte degli ortolani di Viterbo, 1481, Rubr. LVI; Statuta hortulanorum Tuscanae, 1422, Rubr. VII; Statuto dell'arte degli ortolani di Tarquinia, 1379, Cap. XIV).

La estructura del gremio se completaba con los compañeros jurados, es decir, los hortelanos inscritos en el mismo. Eran pequeños propietarios, trabajadores agrícolas y vendedores de frutas y verduras (Statuto dell'arte degli ortolani di Viterbo, 1481, Rubr. LXXVIII; Statuta hortulanorum Tuscanae, 1422, Rubr. XXXII; XXXVII; XXII; Statuto dell'arte degli ortolani, 1379, Capp. V; XXIV).

Según los estatutos de los campesinos de Tarquinia, las condiciones para pertenecer al gremio de agricultores eran:

- Ejercer la actividad agrícola o la relacionada con la actividad comercial de frutas y verduras.

- Residir y poseer el domicilio en Tarquinia (independientemente del lugar de nacimiento).

- Pronunciar el juramento de unión al gremio ante los rectores, realizándolo en latín y con las manos sobre el Evangelio.

- El pago de una cuota de afiliación (Capp. I; V; XXIV; XXXVI; XLVIII; LIV).

En cuanto a la condición de residencia, el lugar de origen del jurado era irrelevante, lo cual se deduce por el texto de los juramentos conservados en el estatuto en el que se leen los nombres de "ortolani" nacidos en Génova, Milán, Florencia, Pisa o incluso en España, con la que de hecho Tarquinia mantenía intensas relaciones comerciales.

En el territorio de Tarquinia existía un puerto al que acudían los barcos de tamaño intermedio, procedentes de Pisa, Génova, Venecia, Sicilia, y también de España y Francia. Desde el puerto se exportaban hierro, cereales y, especialmente, trigo, por ello Tarquinia era conocida como el "granero de Roma" (horreum urbis).

Por otro lado, al puerto llegaban mercancías de todo el Mediterráneo, así como de tierras más remotas, así lo demuestran los fragmentos de cerámica de origen musulmán enterrados cerca del puerto y las mismas páginas del estatuto de los ortolani (hortelanos) se establecía que los honorarios de los oficiales se liquidaban con pimienta, la cual era una preciada mercancía oriental (Società Tarquiniense d'Arte e Storia, 1999, pp. 225-266).

\subsection{Disposiciones sobre la práctica del riego}

Los estatutos dedican gran parte de su exposición a la disciplina de la práctica del riego, tanto desde el punto de vista de la distribución del agua entre los agricultores como desde el punto de vista del mantenimiento de las estructuras hidráulicas. En cuanto a la distribución del agua entre los agricultores, se aplicaban los principios del Derecho común romano, lo cual implicaba que cada agricultor debía utilizar el 
agua que fluía naturalmente en las parcelas agrícolas, sin alterar el curso natural de las acequias $u$ otras obras de riego (Cuturi, pp. 57-58).

A estas normas generales, tomadas del Derecho agrario romano, se añadieron las detalladas disposiciones estatutarias municipales y corporativas que, obviamente, tenían un carácter particular, ya que estaban destinadas a producir efectos en un territorio determinado y, por tanto, a adaptarse a las condiciones morfológicas específicas de esa zona.

En general, el curso del agua y las estructuras para su explotación eran competencia legislativa del municipio, que ciertamente podía conceder el uso o incluso atribuir ámbitos de autonomía decisoria al gremio de agricultores como principal usufructuario de este recurso (Cortonesi, 1988, pp. 15-17).

Los Estatutos de agricultores señalan que el criterio de distribución de los derechos de riego era cronológico, representado por el día natural o litúrgico, método dirigido a evitar las disputas entre usuarios, pero que al mismo tiempo no garantizaba una disponibilidad constante de agua (Statuto dell'arte degli ortolani di Viterbo, 1481, Rubr. XXIX, XXXIII; Statuta hortulanorum Tuscanae, 1422, Rubr. XXXI; Statuto dell'arte degli ortolani di Tarquinia, 1379, Cap.).

Según el capítulo XXXII del estatuto de los agricultores de Tarquinia las tierras situadas en el valle de S. Giovanni se dividían en dos zonas: una situada por encima del camino del mismo valle; y la otra justo por debajo.

En la primera zona se podía regar desde el mediodía hasta la puesta de sol, en la segunda desde la puesta de sol hasta el día siguiente (justo antes del mediodía, cuando comenzaba el turno del otro grupo). En los turnos de riego, los campesinos tomaban de referencia el tañido de las campanas de las iglesias cercanas a las murallas de la ciudad, las cuales sonaban a mediodía.

Los estatutos de Tarquinia (capítulos XXXII y XIX), seguramente para suavizar la rigidez del sistema, preveían en deferencia al carácter solidario de la corporación, que los campesinos de la misma, así como sus familias, pudieran acudir a los huertos de los demás miembros que debían ceder una franja de terreno para que el campesino en dificultades pudiera captar el agua.

En Viterbo supervisaban la distribución del derecho de riego los "balivi viarum et fontium", cuatro de ellos eran los designados bajo la jurisdicción de los rectores de la corporación de los "ortolani". En la ciudad de Tuscania la gestión de las fuentes se limitaba a ciertas estaciones como el verano (Statuto dell'arte degli ortolani di Viterbo, 1481, XXIX, XXXIII; Statuta hortulanorum Tuscanae, 1422, Rubr. XXXI).

\subsection{El mercado semanal}

Como en muchas otras ciudades mercantiles italianas y europeas, en los municipios de Viterbo, Tarquinia y Tuscania existía un mercado diario y semanal, reservado principalmente a los pequeños comerciantes y productores del campo en el que era 
posible comprar productos alimenticios, calzado, ropa, herramientas de hierro y madera, plantas, semillas, animales, objetos usados y productos agrícolas, como frutas, cebollas, ajos, calabazas, pimientos, coles, lechugas y otras hortalizas (Cortonesi, 1988, 1996; Imberciadori, 1983, Greci, 2011; Bonardi, 2003).

El volumen comercial del mercado semanal era de alcance limitado, al estar dirigido a la comunidad local y estar rígidamente regulado por las autoridades municipales y gremiales, que fijaban los precios, los horarios de trabajo, los lugares de la plaza para los puestos y las modalidades de realización de los intercambios comerciales (Cherubini, 1996; Naso, 2014; Tangheroni, 1986; Grohman, 2011; Cavaciocchi, 2001; Ait, 2005).

Las normas detalladas sobre la gestión del mercado figuraban en los estatutos de los gremios de campesinos, a los que se reservaba una considerable autonomía de decisión. En Tarquinia, el mercado semanal se celebraba los miércoles bajo el arco del palacio municipal, los demás días de la semana los agricultores sólo podían vender cidras, limones y naranjas amargas a quienes vivían o tenían una tienda cerca del ayuntamiento, mientras que el domingo no se permitía ninguna actividad comercial (Statuto dell'arte degli ortolani, 1379, Capp. XVII, LVII).

En Viterbo, el día reservado para el mercado semanal era el domingo, la rúbrica LVIII del estatuto de los "ortolani" prohibía a los compañeros jurados salir en la noche del sábado a tomar los mejores puestos o a ocupar más de un puesto del mercado que no podía tener una superficie superior a cuatro pies.

En el estatuto de los "ortolani" de Tuscania, las rúbricas XXXII y XXXVII especifican cómo se asignaba a las mujeres, identificadas con el término pizicarolae, la venta de frutas y verduras. El comercio de hortalizas sólo podía ser ejercido por personas que hubieran prestado juramento al gremio, lo que incluía, por tanto, no sólo a los miembros de la corporación en sentido estricto, sino también, una vez cumplidos los quince años, a sus hijos y a las mujeres encargadas de la venta en la plaza.

El Estatuto de Tarquinia contiene disposiciones precisas destinadas a garantizar el orden durante los días de mercado. En el mercado, a los vendedores o vendedoras no se les permitía llamar a la gente para que se acercara a ellos, ni obstaculizar de ninguna manera los intereses de otros compañeros (Statuto dell'arte degli ortolani, 1379, Capp. XXV, XXX).

Para las mujeres que se dedicaban a la venta, se dictaban disposiciones precisas para evitar que durante el mercado levantaran la voz o lanzaran insultos, estando prohibido de hecho contratar fruteras pendencieras en el mostrador de venta, las cuáles si eran sorprendidas gritando o creando desorden, debían ser expulsadas inmediatamente y no volver a realizar ningún servicio para la corporación por orden de los rectores (Statuto dell'arte degli ortolani, 1379, Cap. LVI). Por último, al anochecer, una vez terminadas todas las actividades del mercado, los vendedores debían limpiar la plaza (Statuto dell'arte degli ortolani, 1379, Cap. XXXIX). 


\subsection{Administración de la justicia}

Los pleitos celebrados ante las autoridades gremiales presentan las características generales del juicio sumario de los estatutos municipales bajo-medievales: en primer lugar, la extrema brevedad y concisión del procedimiento, no lastrado por las formalidades del proceso romano-canónico y por términos excesivamente largos; en segundo lugar, la facultad de definir el juicio según la equidad, ya que el iudex debía atenerse a la objetividad del hecho, sin necesidad de recurrir a incomprensibles elucubraciones jurídicas, sino atendiendo a la concreción del caso y a las necesidades prácticas de los miembros del gremio, al que él mismo pertenecía (Lattes, 1884; Cuturi, pp. 26-27; Belda Iniesta-Coretti, 2016; Sella, 1927; Dani, 2006).

Por tanto, el proceso se caracterizaba por la máxima simplicidad y rapidez, en particular, la sentencia se promovía generalmente sin la presentación de un libelo formal, sino mediante la citación escrita u oral a la que seguía el juicio. Al juez se le reconoció una importante discrecionalidad decisoria al poder interrogar, libremente y sin excesivos formalismos, a los testigos que debían responder puntualmente (Lattes, 1884, pp. 262-264).

A continuación, las partes podían adjuntar las pruebas como escrituras públicas, escritos privados, testimonios, juramentos y confesiones, cuya eficacia era apreciada por el tribunal en virtud de las normas del derecho común y del conocimiento directo de las personas que habían invocado su decisión (Lattes, 1884, pp. 281-286).

El proceso, tras la valoración de las pruebas y las excepciones, llegaba a la sentencia en pocos días, de la que se levantaba acta (conocida como bastardello) y contra la que a menudo se preveía un recurso ante las autoridades municipales que, de nuevo en poco tiempo, debían volver a examinar la causa. Finalmente, si la decisión no era impugnada, se convertía en ejecutiva y otorgaba a los rectores el derecho de emitir un precepto en virtud del cual el deudor era puesto en mora y obligado a pagar en la fecha establecida; si el condenado no cumplía la orden dada, al expirar el plazo establecido, se le embargaban sus bienes. Algunos estatutos preveían, además del procedimiento judicial, el arbitraje confiado a árbitros elegidos entre los inscritos en el gremio o entre los juristas (Lattes, 1884, pp. 294300; Cuturi, 1883, pp. 43-49).

En el caso de los gremios en cuestión, sólo se remitieron a los rectores algunos casos relacionados con la agricultura, que en una primera aproximación coinciden con las materias recogidas en los códigos agrarios. En particular, había dos áreas principales de competencia de los rectores: la regulación del mercado semanal y la regulación del riego, temas que de hecho constituyen la mayor parte de los estatutos (Statuto dell'arte degli ortolani, 1379, Capp. XVII, XXV, XXVI, XXVII, XXVIII, XXX, XXXI, XXXII, XXXIII, XXXIV, XXXV, XXXIX; Statuto dell'arte degli ortolani di Viterbo, 1481, Rubr. XV, XXII, XXIII, XXVIII, XXXV; Statuta hortulanorum Tuscanae, 1422, Rubr. V, XII, XXI, XXIII). 
La vulneración de las disposiciones sobre los dos ámbitos de competencia permitía, por tanto, remitir el asunto al foro corporativo, que en ese caso tenía una competencia casi exclusiva, como se deduce, por ejemplo, del estatuto municipal de Tarquinia, donde no hay ninguna referencia normativa al riego o a la celebración del mercado de frutas y verduras (Statuta Civitatis Corneti, 1545, Liber V, Cap. L). Para todos los demás asuntos que podían surgir en el curso de la actividad agrícola, el tribunal municipal tenía jurisdicción concurrente o exclusiva.

En cuanto al procedimiento ante los jefes del gremio, la acusación podía realizarse mediante citación oral, sin formalidades ni presentación de libelos, por parte de los compañeros jurados o sus familiares.

Durante la fase preliminar del procedimiento sumario, los magistrados de la corporación estaban facultados para utilizar "investigadores" que acudieran a los campos para comprobar y estimar los daños causados a los campesinos. Una vez comprobada la verdad, en base al resultado de las pruebas y a las máximas de experiencia que los rectores, como hortelanos, debían poseer con toda seguridad, se dictaba la sentencia, que los asociados, según las disposiciones expresas del estatuto, estaban obligados a cumplir, sufriendo considerables multas en caso contrario.

En cuanto a las sanciones reguladas en los estatutos, eran principalmente pecuniarias y su cuantía aumentaba en proporción a la reincidencia del infractor hasta llegar a la sanción extrema que suponía la expulsión del gremio con la consiguiente pérdida de todos los derechos relacionados sobre todo con la práctica del riego y el mercado (Statuto dell'arte degli ortolani, 1379, Capp. I, II, III, IV, V, VI, VII, VIII, IX, X; Statuto dell'arte degli ortolani di Viterbo, 1481, Rubr. I, II, III, VI, V, VI, VII, VIII; Statuta hortulanorum Tuscanae, 1422, Rubr. I, III, IV, VIII, X, XIII). La recaudación de las multas se dividía generalmente, salvo algunas variantes, a la mitad que correspondía a la corporación mientras que la parte restante se dividía entre el acusador y los rectores.

\section{EL ARS BOBACTERIORUM URBIS DE ROMA Y SUS ESTATUTOS}

Como ya se ha señalado, entre los gremios agrícolas medievales italianos, el ars bobacteriorum de Roma presenta algunas peculiaridades muy originales que hacen conveniente un estudio autónomo (Ricci, 1893, pp.131-180; Carocci-Vendittelli, 2009, pp. 46-48; Lori Sanfilippo, 2001, pp. 67-72; Vigueur, 2003, p. 219; Decupis, 1906, pp. 36-39).

\subsection{Orígenes y características del ars bobacteriorum y de los estatutos}

Es necesario realizar una aclaración sobre el nombre del gremio romano: ars bobacteriorum. Desde el punto de vista etimológico, en el Glossarium medice et infimce 
latinitatis de Du Cange (1883, col. 683b) parece que el término bobatterius sea de origen galo (Bobaiche) y se refiere genéricamente al arte de la agricultura: Agricola, ad agriculturam pertinens.

De hecho, el ars bobacteriorum no estaba destinado a una categoría específica de trabajadores de la tierra o de propietarios, sino que, como se deduce del proemio de los Estatutos de 1407, se dirigía indistintamente a "cuanto ars ipsa agricolturam continet"1. Una confirmación más de la fuerza general de los estatutos en el sector agrícola proviene del artículo XX de los Estatutos de 1407, que enumera con gran precisión todas las categorías de sujetos sometidos a la jurisdicción del gremio, independientemente de su estatus, rango y dignidad. Esto demuestra cómo en Roma todas las corporaciones de oficios entre quienes ejercían una profesión relacionada con la agricultura estaban sujetas al ars bobacteriorum.

La larguísima y detallada lista del capítulo XX de los Estatutos menciona a los propietarios de tierras, a los propietarios de ganado, a los trabajadores de la tierra nombrados de diversas maneras, a los vendedores de productos agrícolas, de ganado o de leña y a cualquier otra actividad, aunque esté indirectamente relacionada con la agricultura ${ }^{2}$.

Una confirmación más de la validez general de los Estatutos puede deducirse del hecho de que en la segunda mitad del siglo XVI, no es posible conocer con certeza la fecha, los Statuta bobacteriorum cambiaron su título por el de Statuta artis agriculturae, nombre que identificaba mejor el objeto de la normativa estatutaria.

1 Premissa debita meditatione pensantes ac mature considerantes quod si cuique convenit debitum modum dare quod homines sub iuris et equitatis regulis gubernentur, ac per omnia sine alterius iniuria vi vant, tanto presidentibus in Arte ipsa convenientius est modum hu iusmodi adhibere, quanto Ars ipsa agriculturam continet.

2 Item, quod illi intelligantur esse de Arte bobacteriorum qui aliquod exercitium de exercitiis dictae Artis et ad ipsam Artem a pertinentibus fecerint seu administraverint per se vel alios eorum nomine. Item et illi qui aliquam possessionem vel casale seu pe dicam aut valsolam terre pro seminando aut silvam, nemus, pan tanum, vel pratum, aut quaecumque animalia quadrupedia habue «rint: nec non omnes et singuli illi qui eorum opera et labores impenderint in aliquo exercitio dictae Artis, videlicet seminando «quodcumque genus bladorum, leguminum et frumentorum, autali cuius ipsorum colendo, et cultivando, terrae quomodolibet pro preditis, mundando, seu metendo aut falciando, coadunando, tra «gliando, aut defendendo blada, legumina aut frumenta supradicta animalia tenendo, aut custodiendo, ipsaque vel aliquod eorundem aut lanam, caseum seu quoscumque alios fructus animalium aut casalium et possessionem praedictorum: emendo aut vendendo, aut «quodvis aliud exercitium in praedictis et circha praedicta faciendo. Itaque omnes et singuli qui in praedictis et circa praedicta et in dependentibus ab eisdem aliquod exercitium fecerint etiam casarole «quae caseum a bobacteriis emerent, nec non bubulci, vaccharii, bufalarii, carrarii, iumentarii, garzarii pecudum, caprarii, porcarii, casegni «seu asinarii,mulatterii, buttarii, et alii quicumqueanimalium pastores et custodes quocumque nomine nuncupati, mundatores, seminatores, stirpatores, messores, adunatores, tragliatores, furcinatores, et veturales frumentorum aut bladorum seu straminum quorumcumque et tenimentorum, cultores de Arte praedicta esse omnino * praedictis quomodolibet declinare, immo foro et iurisdictioni curie «Artis praedictae occasioni dictarum rerum et cuiuslibet ipsarum a intelligantur omnino esse suppositi et subiecti: non obstante quod a ad dictam Artem forte non solverunt, aut in libro hominum dictae «Artis scripti et adnotati non essent, ac aliis quibuscumque in con a trarium editis non obstantibus, quibus auctoritate et potestate praedictis derogaverunt et voluerunt esse praesentibus derogatum. 
En cuanto al texto de los Estatutos, la versión más antigua que ha llegado hasta nosotros es la de 1407, seguida de las ediciones impresas de 1526 y 1566, las reimpresiones de 1573 y 1627 y, por último, la versión traducida al italiano de 1718. En los Estatutos de 1407 se hace referencia a los borradores anteriores de los estatutos, a las antiguas ordinationes artis, así como a las consuetudines remotas que luego se reunieron de forma más ordenada en el Estatuto de 1407 (Ricci, 1893, pp. 141-142).

Todo ello demuestra, por tanto, que el gremio de los agricultores romanos era mucho más antiguo que el del 1407, hecho confirmado por diversos testimonios históricos que atestiguan su existencia a partir de la segunda mitad del siglo XIII, periodo en el que la asociación ya contaba con un sistema jurídico propio representado por los estatuta, ordinationes y consuetudines recordadas por la versión de 1407 (Statuta bobacteriorum urbis, 1407, capp. 7, 47).

Entre los documentos que atestiguan la existencia del gremio antes de 1409, el más significativo es la carta del papa Urbano V al senador de Roma en 1368, en la que se lee que incluso el propio Papa fue elegido cónsul de la corporación agrícola, circunstancia que demuestra el prestigio y la importancia económico-política del ars bobacteriorum urbis al que se inscribían las familias romanas más ricas y nobles como propietarias de los vastos feudos de la ciudad eterna (Ricci, 1893, p. 158).

En el siguiente apartado se tomarán como referencia los Estatutos de 1407 para realizar el estudio.

\subsection{Disposiciones disciplinarias y jurisdicción}

Desde el punto de vista del contenido, los Estatutos presentan tres núcleos temáticos:

- el primero regula los cargos asociativos;

- el segundo aborda las competencias jurisdiccionales de la corporación;

- el tercero contiene las normas más propiamente vinculadas al ejercicio de la actividad agraria.

En la primera parte, no hay diferencias notables con respecto a los estatutos ya examinados y, en general, con respecto a los estatutos gremiales medievales, salvo variaciones irrelevantes en la terminología. Por ejemplo, en lugar de los rectores, en la cúspide de la asociación se sitúan los cónsules, seguidos, como es habitual, de los demás cargos: el tesorero, los consejeros y el notario.

Sin embargo, en las asociaciones examinadas en el párrafo anterior no se preveía la figura del asesor consulum que, según el capítulo 4 de los Statuta bobacteriorum, debía ser un doctor en derecho al que los cónsules debían pedir un dictamen jurídico antes de dictar sentencia en los casos más complejos; además, el asesor debía defender los derechos del gremio y, en ausencia de los cónsules, podía impartir justicia en los litigios sometidos a la jurisdicción del gremio. 
En este sentido, la jurisdicción de la corporación era muy amplia e incluía todos los litigios surgidos en el ejercicio de la actividad agrícola o ganadera con independencia de que las partes fueran o no miembros de la corporación: de hecho, estaban sometidos al tribunal de los cónsules todos los ciudadanos romanos que hubieran participado en alguna actividad agrícola (Statuta bobacteriorum urbis, 1407, capp. $23,24,31,68)$.

En particular, el gremio tenía competencia exclusiva sobre los litigios relativos al cultivo de los campos, que debía realizarse según las mejores prácticas agrícolas, los contratos de arrendamiento de tierras concedidas ad laborandum (art. 51, 54) o de animales dados ad soccitam (Capp. 95, 96, 97) ad custodiendum et subcreandum (art. 68), los pleitos sobre los límites entre parcelas (art. 62) y los daños causados por el ganado o los hombres. En este último sentido eran castigados los que habían cortado los árboles de la tierra recibida en arrendamiento (art. 56), prendido fuego a los rastrojos antes de la fiesta de la Asunción (art. 84), quitado la corteza de los árboles (art. 88) o sacado el trigo sin el permiso del propietario de la tierra (art. 54).

Los infractores de las normas del gremio debían comparecer ante el tribunal del gremio presidido por los cónsules, o en su lugar por los asesores, y en caso de perder una parte de la indemnización se debía a la Cámara de la asociación (artt. 69-77).

Los casos reservados a la competencia del ars bobacteriorum urbis sólo podían ser tratados en los días establecidos (dies iuridici) y en las horas determinadas (horae iuridicae causarum) según las características del proceso sumario ya descritas para los gremios medievales (cap. 39). La decisión de los cónsules era definitiva e inapelable; la parte perdedora, en caso de considerar exagerada la sentencia, sólo podía pedir la anulación o modificación de la misma a uno o dos peritos pertenecientes al ars bobacterirorum cuyo dictamen cerraba definitivamente la cuestión sin posibilidad de réplica (cap. 41).

\section{FUENTES PRIMARIAS}

Statuto dell'arte degli ortolani di Corneto, 1379, en Archivio Storico, Tarquinia; produzione seriale, 300 .

Statuta hortulanorum Tuscanae, 1422, en Archivio comunale, Tuscania.

Statuto dell'arte degli ortolani di Viterbo, 1481, en Biblioteca degli Ardenti, Viterbo; Cat. n. II. E. III. 34.

Statuta Civitatis Corneti, 1545, en Archivio Storico, Tarquinia, produzione seriale, 302.

Albericus De Rosate, De statutis, I, 6.

BALDI, ad D. 1, 1 de iust. Et iure, 1.9 omnes populi n. 32.

BALDI, ad C. 4, 18 de const. pec. 1. 2 recepticia n. 9.

BARTOLI, ad D. 1,1 de iustitia et iure, 1.9 omnes populi, n. 6 . 


\section{REFERENCIAS BIBLIOGRÁFICAS}

Arangio Ruiz, V. (2006). Istituzioni di diritto romano. Napoli: Casa Editrice Dott. Eugenio Jovene.

ARTIFONI, E. (1988). Forme del potere e organizzazione corporativa in età comunale: un percorso storiografico. En C. Mozzarelli, Economia e corporazioni. Il governo degli interessi nella storia d'Italia dal medioevo all'età contemporanea (pp. 9-40). Milano: Giuffrè.

Belda Iniesta, J., Coretti, M. (2016). Le Clementine Dispendiosam e Saepe Contigit come paradigma di sommarietà. Alcune note in chiave utroquistica. Monitor Ecclesiasticus. Commentarius internationalis iuris canonici, (131.2), 360-423.

Bellomo, M. (2016). L'europa del diritto comune. La memoria e la storia. Leonforte: Euno Edizioni.

BeStA, E. (1925). Fonti: legislazione e scienza giuridica. Dalla caduta dell'impero romano al secolo decimo sesto. Milano: Hoepli.

BEZzINA, D. (2013). Organizzazione corporativa e artigiani nell'Italia medievale. Reti Medievali Rivista, 14 (1), 351-374. https://doi.org/10.6092/1593-2214/391.

BlaCK A. (1990). Community in Historical Perspective. A translation of selections from Das Deutsche Genossenschaftsrecht (the German Law of Fellowship) by Otto von Gierke. Cambridge: Cambridge University Press. https://doi.org/10.1017/ CBO9780511521386

Black, A. (1984). Guilds and Civil Society in European Political Thought from the Twelfth Century to the Present. New York: Cornell University Press.

Braunstein, Ph. (2003). Travail et entreprise au moyen âge. Bruxelles: De Boeck.

Braunstein, Ph. (2007). Production et travail dans les villes à la fin du moyen âge. História: revista da faculdade de letras da universidade do Porto, (8), 11-23.

Calasso, F. (1954). Medioevo del diritto. $I^{\circ}$ - Le fonti. Milano: Giuffrè.

CAlasso, F. (1965). Gli ordinamenti giuridici del rinascimento medievale. Milano: Giuffrè.

Campitelli, A. (1993). Europeenses. Presupposti storici e genesi del diritto comune. Bari: Cacucci.

CANNING, J. (1980). The corporation in the political thought of the Italian jurists of the thirteenth and fourteenth century. History of political thought, (1), 9-32.

Carocci, S., Vendittelli, M. (2009). Casali, castelli e villaggi della campagna romana nei secoli XII e XIII. En P. Delogu, A. Esposito (Eds.), Sulle orme di Jean Coste: Roma e il suo territorio nel tardo medioevo (pp. 37-51). Roma: Viella.

Caroselli, M. R. (1957). Corporazione medioevale. En A. Azara, E. Eula (eds.), Novissimo Digesto Italiano (vol. IV, p. 864). Torino: Uninoe tipografico-editrice torinese.

COORNAERT, E. (1947). Les ghildes médiévales (Ve-XIVe siécles): définition-évolution. Revue historique, (199), 208-243. 
Cortonesi, A., Viola, F. (2006). Le comunità rurali e i loro statuti (secoli XII-XV). Atti dell'VIII Convegno del Comitato italiano per gli studi e le edizioni delle fonti normative, Viterbo 30 maggio- $1^{\circ}$ giugno 2002. Roma.

CRISTOFERI, D. (2016). Da usi civici a beni comuni: gli studi sulla proprietà collettiva nella medievistica e nella modernistica italiana e le principali tendenze internazionali. Studi Storici, vol. 57, (3), 577-604.

DANI, A. (2006). Il processo per danni nello Stato della Chiesa (secoli XVI-XVIII). Bologna: Monduzzi.

DANI, A. (2012). Gli statuti comunali nello Stato della Chiesa di Antico regime: qualche annotazione e considerazione. Historia et ius, (2), 1-14.

DANI, A. (2013). Le risorse naturali come beni comuni. Arcidosso: Effigi.

De Robertis, F. M. (1971). Storia delle Corporazioni e del Regime Associativo nel mondo Romano. Bari: Adriatica Editrice.

DE RoberTis, F. M. (1981). Il fenomeno associativo nel mondo romano. Dai collegi della Repubblica alle corporazioni del basso impero. Roma: L'erma Di Bretschneider.

DECUPIS, C. (1906). Per gli usi civici nell'agro romano: e nella provincia di roma contributo storico. Rivista Internazionale di Scienze Sociali e Discipline Ausiliarie, (157), 34-61.

DEGRASSI, D. (1996). L'economia artigiana nell'Italia medievale. Roma: Carocci.

DEGRASSI, D. (2000). Organizzazioni di mestiere, corpi professionali e istituzioni alla fine del medioevo nell'Italia centro settentrionale. En M. Meriggi, A. Pastore, Le regole dei mestieri e delle professioni, secc. XV-XIX (pp. 17-35). Milano: Franco Angeli.

DEGRASSI, D. (2001). Gli artigiani nell'Italia comunale. En Centro italiano di studi di storia e d'arte (ed.), Ceti, modelli, comportamenti nella società medievale (secoli XIII-metà XIV), Atti del diciassettesimo convegno internazionale di studi (pp. 147173). Pistoia: Viella.

Degrassi, D. (2010). Il mondo dei mestieri artigianali. En S. Carocci (Ed.), La mobilità sociale nel Medioevo (pp. 273-287). Roma: École française de Rome.

Diosono, F. (2007). Collegia. Le associazioni professionali nel mondo romano. Roma: Edizioni Quasar.

DONDARINI, R. (2006). Comunità rurali: beni comuni e beni collettivi. En A. Cortonesi, F. Viola (Ed.), Le comunità rurali e i loro statuti (secoli XII-XV). Atti del VIII Convegno del Comitato italiano per gli studi e le edizioni delle fonti normative (pp. 115-132). Roma: Gangemi editore.

Du CANGE, C. (1883-1887). Glossarium mediae et infimae latinitatis, t. 1, col. 683b. Niort: L. Favre.

FERRERI, T. (2018). Istituzioni e governo del territorio nello Stato pontificio: ricerche sul Patrimonio di San Pietro in Tuscia (secoli VI-XIII). Historia et ius, (14), 1-42. 
Flambard, J.M. (1981). Collegia compitalicia: phénomène associatif, cadres territoriaux et cadres civiques dans le monde romain à Tépoque républicaine. Ktèma, (6), 143-166. https://doi.org/10.3406/ktema.1981.1842

Giontella, G. (1971-1972). Gli statuti degli ortolani di Tuscania del 1422. Annali della Libera Università della Tuscia, anno III, fascicoli I-IV.

Greci, R. (1995). Le corporazioni. Associazioni di mestiere nell'Italia del medio evo. Storia e dossier, (99), 61-106.

KlopPenBORG, J. S., Wilson, S. G. (2002). Voluntary Associations in the Graeco-Roman World. Milton Park: Taylor \& Francis. https://doi.org/10.4324/9780203216705

LATTES, A. (1884) Il diritto commerciale nella legislazione statutaria delle città italiane. Studi di Alessandro Lattes. Milano: U. Hoepli.

LEICHT, P. S. (1937). Storia del diritto italiano. Le fonti, lezioni. Milano: Giuffrè.

LORI SANFILIPPO, I. (2001). La Roma dei romani: arti, mestieri e professioni nella Roma del Trecento. Roma: Istituto storico italiano per il Medio Evo.

Maire Vigueur, J.-C. (2003). Des brebis et des hommes. La transhumance à Rome à la fin du moyen âge En D. Barthélemy, J.-M. Martin, Liber largitorius. Études d'histoire médiévale offertes à Pierre Toubert par ses élèves (pp. 219-237). Genève: Droz.

Mennella, G., Apicella, G. (2000). Le corporazioni professionali nell'Italia romana: un aggiornamento al Waltzing. Napoli: Arte tipografica.

MinnuCCI, G. (2017). La donna giudice, Innocenzo III e il sistema del diritto comune. Vergentis: revista de investigación de la Cátedra Internacional conjunta Inocencio III, (4), 77-106.

Montanos FerRín, E. (2007). La herencia de Francesco Calasso: actualidad de una perspectiva innovadora. Consideraciones para un perfil de historia de la historiografía europea. Anuario de historia del derecho español, (77), 469-479.

OcCHIPINTI, E. (1990). Quarant'anni di studi italiani sulle corporazioni medievali tra storiografia e ideologia. Nuova rivista storica, 74, 101-174.

ParaVicini Bagliani, A. (2010). Il papato nel secolo XIII. Cent'anni di bibliografia (1875-2009), Firenze: Edizioni del Galluzzo.

PATTERSON, J. (1993). Patronage, collegia and burial in imperial Rome. En S. Bassett (ed.), Death in towns: urban responses to the dying and the dead (pp. 15-27). Leicester: Leicester University Press.

PATTERSON, J.R. (1994). The collegia and the transformation of Italian towns. En LItalie d'Auguste à Dioclétien. Actes du colloque international de Rome (pp. 227238). Roma: Publications de l'École française de Rome.

PinI, A.I. (1976). L'associazionismo medievale: comune e corporazioni. Bologna: Cooperativa Libraria Universitaria Editrice Bologna.

Pini, A.I. (1986). Città, comuni e corporazioni nel medioevo italiano. Bologna: Cooperativa Libraria Universitaria Editrice Bologna. 
RASI, P. (1940). Le corporazioni fra gli agricoltori. Studio storico-giuridico. Milano: Giuffrè.

RiccI, G. (1893). la Nobilis universitas bobacteriorum Urbis. Archivio della Società romana di Storia Patria, (16), 131-180.

Rosati, S. (2013-2014). Lo statuto degli ortolani di Corneto del 1379. Studio storicogiuridico. Bollettino della Società tarquiniense d'arte e storia, (40), 137-171.

ROSATI, S. (2018). The medieval agricultural corporations in the territories of the patrimony of Saint Peter. Historical-juridical study (centuries XIV-XV). En A. Katancevic, M. Vukotic, S. Vandenbogaerde, V. M. Minale (Eds.), History of legal sources. The changing structure of Law (pp. 165-173). Belgrade: University of Belgrade.

Schioppa, A. P. (1964). Giurisdizione e statuti delle arti nella dottrina del diritto comune. Studia et documenta historiae et iuris, (30), 179-234.

Sella, P. (1927). Il procedimento civile nella legislazione statutaria italiana. Milano: Hoepli.

Società Tarquiniense d'Arte e Storia (1999). I pellegrini nella Tuscia medioevale: vie, luoghi e merci; atti del convegno di studi, 4 - 5 ottobre 1997. Tarquinia: Tipografia Giacchetti.

Sprandel, R. (1991). Corporations et luttes sociales au temps préindustriel. En A. Guarducci, Forme ed evoluzione del mondo del lavoro in Europa secc. 13-18. Atti della tredicesima settimana di studio 2-7 maggio 1981 (pp. 353-366). Firenze: Le Monnier.

TAMASSIA, N. (1898). Le associazioni romane nel periodo precomunale. Archivio giuridico Filippo Serafini, (66), 112-141.

Thrupp, S. (1965). The Gilds. En M. Postan, E. Rick, and M. Miltey (eds.), Cambridge Economic History of Europe (pp. 230-280), Vol. III. Cambridge: Cambridge University Press). https://doi.org/10.1017/CHOL9780521045063.006

Wolf, A. (1993-1994). Los iura propria en europa en el siglo XIII. Glossae. revista de historia del derecho europeo, (5-6), 35-41.

Zanoboni, M.P. (2009). Salariati nel Medioevo (secoli XIII-XV). Ferrara: Nuove Carte. 